\title{
Review
}

\section{Falls Self-Management Interventions for People with Parkinson's Disease: A Systematic Review}

\author{
Charlotte L. Owen ${ }^{\mathrm{a}, \mathrm{b}, *}$, Kinda Ibrahim ${ }^{\mathrm{a}, \mathrm{b}}$, Laura Dennison ${ }^{\mathrm{c}, 1}$ and Helen C. Roberts ${ }^{\mathrm{a}, \mathrm{b}, 1}$ \\ ${ }^{a}$ Academic Geriatric Medicine, University of Southampton, Southampton, UK \\ ${ }^{\mathrm{b}}$ National Institute for Health Research Collaboration for Leadership in Applied Health \\ Research and Care (NIHR CLAHRC) Wessex, University of Southampton, Southampton, UK \\ ${ }^{\mathrm{c}}$ School of Psychology, University of Southampton, Southampton, UK
}

Accepted 13 February 2019

\begin{abstract}
.
Background: Falls are common in Parkinson's disease (PD). Increased involvement of people with Parkinson's (PwP) in their care has been associated with enhanced satisfaction. Self-management programmes in other long-term conditions (LTCs) have led to improvements in physical and psychological outcomes. These have been more effective when targeted toward a specific behavior.

Objective: This paper aimed to identify and review falls self-management interventions for PwP.

Methods: A systematic review was conducted. Electronic databases were searched in June 2018. Primary research studies (any design) reporting the delivery of falls self-management interventions to PwP were included. Data was extracted from each article and synthesised narratively.

Results: Six articles were identified, relating to five different self-management interventions. All described a self-management intervention delivered alongside physiotherapy. Intervention delivery was through either group discussion $(n=3)$ or falls booklets $(n=3)$. Interventions were often incompletely described; the most common components were information about the condition, training/ rehearsal for psychological strategies and lifestyle advice and support. Arising from the design of articles included the effects of self-management and physiotherapy could not be separated. Three articles measured falls, only one led to a reduction. Four articles measured quality of life, only one led to improvement. No articles assessed skill acquisition or adherence to the self-management intervention.

Conclusions: Few falls self-management interventions for PwP have been evaluated and reported. The components of an effective intervention remain unclear. Given the benefits of self-management interventions in other LTCs, it is important that falls self-management interventions are developed and evaluated to support PwP.
\end{abstract}

Keywords: Accidental falls, parkinson's disease, patient education as topic, review, self care

\section{INTRODUCTION}

Falls are common in Parkinson's disease (PD), with two thirds of people with Parkinson's (PwP)

\footnotetext{
${ }^{1}$ These authors jointly led this project.

*Correspondence to: Charlotte L. Owen, Academic Geriatric Medicine, E Level Centre Block Mailpoint 807, Southampton General Hospital, Southampton, Hampshire, SO16 6YD, UK. Tel.: +442381 206128; E-mail: c.l.owen@ soton.ac.uk.
}

falling each year compared to one third of the general older population [1]. Falling often leads to a fear of falling and a decline in physical activity, which is associated with negative outcomes including a decline in both physical function and quality of life (QOL) $[2,3]$. Falling in PD is multifactorial; specific risk factors include freezing of gait and postural instability but recurrent falls are more likely among those with cognitive impairment $[4,5]$. The preva- 
lence of cognitive impairment rises with increasing patient age and disease duration but mild dysfunction has been reported in 24\% of newly diagnosed PwP $[6,7]$. The majority of PwP live in their own homes helped by family members, 'informal caregivers', who provide vital physical, emotional, and social support $[4,8]$. Caregivers of PwP often feel unprepared and unsupported in this role, and the onset of falling has been associated with increased caregiver burden $[9,10]$.

Systematic reviews of physiotherapy interventions for falls have reported inconclusive results, which may relate to the nature of the studies included. These reviews have been based upon studies of predominantly low-moderate methodological quality with heterogeneity of interventions and outcomes measured [11-13]. One recent systematic review conducted by Shen et al. reported a reduction in fall rate, however, the effect size was small and outcomes from individual studies were mixed [12]. Whereas, earlier reviews conducted by Allen et al. and Tomlinson et al., reported no effect of physiotherapy on fall rate or the proportion of fallers respectively $[11,13]$. In Shen et al. the greatest benefits were observed in association with facility based training [12]. By design, these studies may exclude PwP with greater disease severity or cognitive impairment, as travel may be more difficult. Rehabilitative interventions may be less effective in those with more advanced PD, for whom falling is more common [14]. Similarly, in a recent large multi-centre randomized controlled trial (RCT), a four month high-intensity structured exercise programme did not lead to improvements in fall rate or QOL in people with mild to moderate dementia or their caregivers [15].

The review of physiotherapy interventions by Tomlinson et al. reported a lack of effect on both fear of falling and quality of life [11]. Whilst a review of occupational therapy based interventions for PwP reported a positive short-term effect on QOL, this was based upon the results of three small studies [16]. This finding was not replicated in two subsequent large RCTs of occupational therapy; in these studies the majority of participants had mild-moderate $\mathrm{PD}$, the very group in whom rehabilitative interventions have been proposed to be more effective $[14,17,18]$.

PwP and their caregivers have expressed a wish for increased involvement and participation in their care, which has been associated with enhanced patient satisfaction [8, 19, 20]. Self-management has been proposed as a potential strategy to help PwP and their caregivers to identify and address problems relating to how PD affects them [21]. Self-management can be broadly defined as increased responsibility or confidence of an individual in managing a long-term condition (LTC) [22]. Self-management programmes in LTCs such as diabetes, stroke, and chronic obstructive pulmonary disease have led to improvements in physical and psychological outcomes [23-27]. Similarly, promoting active patient engagement, has been shown to be cost effective by improving quality of care and medical outcomes in older patients with multimorbidity [28].

The majority of self-management programmes are based on Bandura's self-efficacy theory which hypothesises that an individual's self-perceived ability to perform a task, or to manage a situation, is directly related to their subsequent likelihood of success [22]. For an individual to self-manage a LTC they might require training in medical, behavioral, and emotional techniques. Arising from the heterogeneity of falls in PD, PwP and their caregivers are likely to differ in what they require to for effective self-management $[4,5]$. Important factors might be knowledge of the contributing risks to falls and skills and confidence in how to modify them, and understanding when and who to contact if difficulties arise.

A 2017 integrative review of self-management programmes for PwP to support any aspect of PD concluded that there was insufficient evidence to support their effectiveness [29]. Following self-efficacy theory, self-management programmes in other LTCs have been more effective when targeted toward a specific behavior as opposed to providing generic advice and support [22, 30]. In this paper we aimed to systematically identify and review falls self-management interventions for PwP, and if possible, assess their efficacy for improving patient and caregiver outcomes, QOL and psychological outcomes. Such information will be beneficial to inform the development of falls self-management interventions for $\mathrm{PwP}$ in research and routine clinical practice.

\section{MATERIALS AND METHODS}

This review was conducted according to the Preferred Reporting Items for Systematic Reviews and Meta-analyses (PRISMA) guidelines [31]. The review protocol was registered on PROSPERO, CRD42017052585. 


\section{Searching strategy and identification of articles}

Searches were conducted in June 2018. Searches aimed to locate all papers relating to selfmanagement in PD. A sensitive and inclusive searching strategy using Medical Subject headings (MeSH) and key free text terms pertaining to (i) PD and (ii) self-management was developed. Animal studies were excluded and the search was limited to studies published since the emergence of the concept of self-management within the literature (1986 onwards) [23]. The search terms were developed in MEDLINE and adapted for use in EMBASE, CINAHL, AMED, PSYCHInfo and Science Citation Index Expanded (Web of Science) (for details about search terms used see Supplementary Table 1). Given the heterogeneity of interventions that may support self-management in LTCs, we did not stipulate that authors needed to use the term 'self-management' [23-26]. The search strategy contained no fallsrelated terms to allow identification of studies where falling was only discussed in the full text. Additional searches were made in clinical trials registers. The grey literature was searched at www.opengrey.eu.

Articles were eligible for inclusion where they reported interventions aimed to improve falls self-management in PwP. Studies were included regardless of design. A broad definition of selfmanagement was used; studies that aimed to increase the responsibility or confidence of $\mathrm{PwP}$ in managing falls. Articles were excluded where the intervention consisted of physiotherapy alone.

Multi-modal interventions were included where one component aimed to support self-management of falling. No restrictions on language were applied.

After removal of duplicates, article titles were screened by $\mathrm{CO}$ for relevance. $\mathrm{CO}$ and $\mathrm{KI}$ independently screened all abstracts for eligibility. Full texts of all potentially relevant articles were reviewed for relevance and discrepancies were resolved through mutual discussion. Reference lists of relevant articles were screened. Figure 1 depicts the search results and inclusion/exclusion process.

\section{Data analysis and assessment of study quality}

Self-management programmes are often complex and multi-faceted. Complex interventions are comprised of multiple interacting components, with additional dimensions to include heterogeneity of those delivering or receiving the intervention [32].
In the wider LTC literature, inadequately detailed reporting of the content and delivery of complex interventions, and the lack of a shared language for describing their components, has been identified as a barrier to drawing clear conclusions about what works and why and to wider implementation [33-35]. The 'Practical Reviews in Self-management Support' (PRISMS) taxonomy was used to characterize the self-management component of each intervention [33]. This tool (Table 1) details 14 components that a self-management programme might contain and has successfully been used to identify the key features of self-management interventions to support patients with diabetes, heart failure, asthma, chronic obstructive pulmonary disease, and cancer $[26,28]$.

$\mathrm{CO}$ extracted data from the selected articles into a predefined database, with all content confirmed by KI. Authors of all included articles were contacted for clarification or to request additional information. $\mathrm{CO}$ and KI independently appraised each included article using the Physiotherapy Evidence Database (PEDro) Scale, an eleven item scale widely used in physical therapy to assess the quality of RCTs [36, 37]. Items on the PEDro scale largely relate to design biases [36, 37]. Ten of the eleven items contribute to the summary score; a summary score of greater than five or six indicates adequate quality (Supplementary Table S2) $[36,37]$. All articles were included irrespective of the outcome of the quality assessment to provide a comprehensive review of the literature. The analysis took the form of a narrative synthesis.

\section{RESULTS}

\section{Article selection and quality appraisal}

Of the 11,693 articles identified, 48 full text articles were screened and four met the eligibility criteria for inclusion. A further two articles were identified through reference screening.

A conference abstract was identified that described the development of a falls self-management guide for PwP to be delivered alongside physiotherapy [38]. The author was contacted; no details of the participants or how the intervention was implemented or evaluated were available, and it was excluded from our review.

No additional records were identified through searches of the grey literature and clinical trials registers. Thus six articles were included in the review (Fig. 1). 


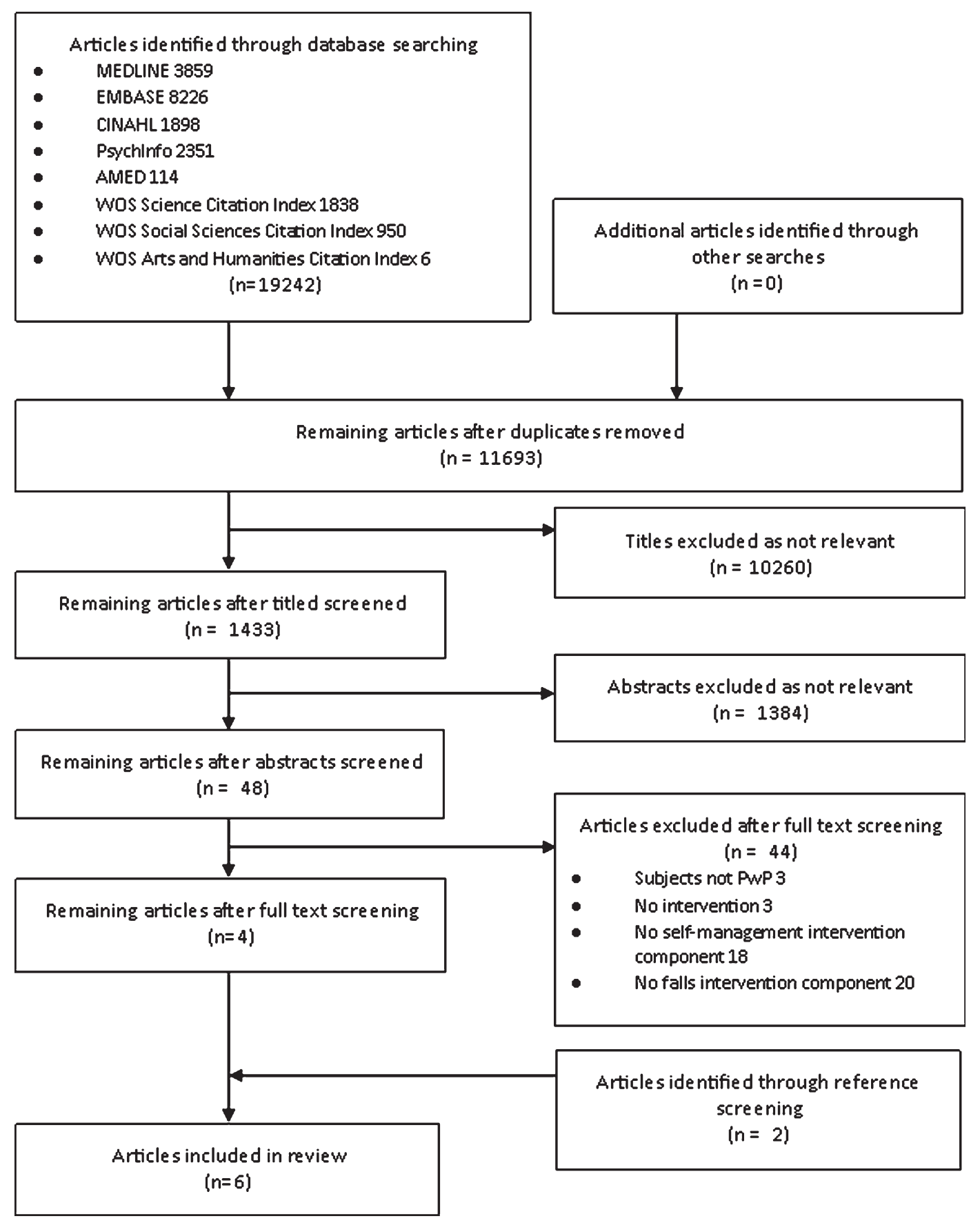

Fig. 1. Preferred Reporting Items for Systematic Reviews and meta-analyses (PRISMA) diagram detailing the search process undertaken in this review. n, number; PRISMA, Preferred Reporting Items for Systematic Reviews and Meta-analyses; PwP, People with Parkinson's.

Five of six articles had summary scores of greater than five out of ten on the PEDro scale, deeming them adequate quality [37, 39-43]. The article by Peteet scored four out of ten, deeming it low quality [44] (Table 3 and Supplementary Table 2).

\section{Article characteristics}

\section{Study design}

The included articles comprised five research papers and one thesis (Table 2). All reported RCTs. Two articles were based on the same population 
Table 1

Practical reviews in self-management support (PRISMS) self-management components of the interventions

\begin{tabular}{|c|c|c|c|c|c|c|}
\hline $\begin{array}{l}\text { PRISMS taxonomy } \\
\text { component }\end{array}$ & Peteet [44] & $\begin{array}{l}\text { Tickle Degnen } \\
\text { et al. [42] }\end{array}$ & $\begin{array}{l}\text { White } \\
\text { et al. [43] }\end{array}$ & $\begin{array}{l}\text { Canning } \\
\text { et al. [41] }\end{array}$ & $\begin{array}{c}\text { Morris et al. } \\
2015 \text { [39] }\end{array}$ & $\begin{array}{l}\text { Morris } \\
\text { et al. [40] }\end{array}$ \\
\hline $\begin{array}{l}\text { A1. Information about the condition } \\
\text { and/or its management }\end{array}$ & $\checkmark$ & & & $\checkmark$ & $\checkmark$ & $\checkmark$ \\
\hline $\begin{array}{l}\text { A2. Information about available } \\
\text { resources }\end{array}$ & & & & $\checkmark$ & $\checkmark$ & $\checkmark$ \\
\hline \multicolumn{7}{|l|}{$\begin{array}{l}\text { A3. Provision of/ agreement on specific } \\
\text { clinical action plans and/or rescue } \\
\text { medication }\end{array}$} \\
\hline A4. Regular clinical Review & $\checkmark$ & & & & & \\
\hline $\begin{array}{l}\text { A5. Monitoring of condition with } \\
\text { feedback }\end{array}$ & $\checkmark$ & & & & & \\
\hline \multicolumn{7}{|l|}{$\begin{array}{l}\text { A6. Practical support with adherence } \\
\text { (medication or behavioral) }\end{array}$} \\
\hline \multicolumn{7}{|l|}{ A7. Provision of equipment } \\
\hline \multicolumn{7}{|l|}{$\begin{array}{l}\text { A8. Provision of easy access to advice or } \\
\text { support when needed }\end{array}$} \\
\hline $\begin{array}{l}\text { A9. Training/ rehearsal to communicate } \\
\text { with health care professionals }\end{array}$ & & & & $\checkmark$ & $\checkmark$ & $\checkmark$ \\
\hline $\begin{array}{l}\text { A10. Training/ rehearsal for everyday } \\
\text { activities }\end{array}$ & & $\checkmark$ & $\checkmark$ & $\checkmark$ & $\checkmark$ & $\checkmark$ \\
\hline $\begin{array}{l}\text { A11. Training/ rehearsal for practical } \\
\text { self-management.Includes skill } \\
\text { acquisition }\end{array}$ & & & & $\checkmark$ & $\checkmark$ & $\checkmark$ \\
\hline $\begin{array}{l}\text { A12. Training/ rehearsal for } \\
\text { psychological strategies Includes } \\
\text { action planning and goal setting }\end{array}$ & $\checkmark$ & $\checkmark$ & $\checkmark$ & $\checkmark$ & $\checkmark$ & $\checkmark$ \\
\hline A13. Social support & $\checkmark$ & $\checkmark$ & $\checkmark$ & & & \\
\hline $\begin{array}{l}\text { A14. Lifestyle advice and support } \\
\text { Includes advice about exercise and a } \\
\text { healthy diet }\end{array}$ & & $\checkmark$ & $\checkmark$ & $\checkmark$ & $\checkmark$ & $\checkmark$ \\
\hline
\end{tabular}

and intervention; the participants in White et al. were a subsample of those from Tickle-Degnen et al. but different outcome measures were reported [42, 43].

No articles were identified that studied the effect of a falls self-management intervention in isolation. Each article evaluated the effect of a self-management intervention alongside a physiotherapy intervention. Canning et al. studied the effect of an intervention that contained physiotherapy and self-management, and compared it to self-management alone [41]. The other five articles studied the effect of an intervention that contained physiotherapy and self-management and compared it to a control which did not contain either of these constituents [39-43].

\section{Setting and participant characteristics}

Trials were conducted between 2003 and 2016; three in Australia and three in the USA. The total number of participants was 718 (range 27 to 231 in each study). Participants were all PwP, no interventions included caregivers. Mean age varied from 58 to 71.4 years; mean time from diagnosis was 5.1 to
8.8 years. In five of six articles, the majority of participants had a Hoehn and Yahr stage of less than three, indicating reduced fall risk [39, 40, 42-44]. All articles excluded those with cognitive impairment. In the three data sets that reported baseline falls, 55 to $78 \%$ had fallen within the last year [39-41].

\section{Intervention delivery}

Self-management interventions were delivered through either group discussion or the provision of falls booklets. In each of the group interventions, falls self-management was embedded within a programme that targeted self-management of a range of PD related difficulties [42-44]. In the three articles containing falls booklets, falls was the key target of the intervention [39-41].

\section{Group discussion}

Three articles, evaluating two different interventions described physical therapist facilitated group discussion of self-management alongside physiotherapy [42-44]. Peteet assessed the effect of a weekly physical exercise and self-management education programme compared to exercise alone [44]. The 


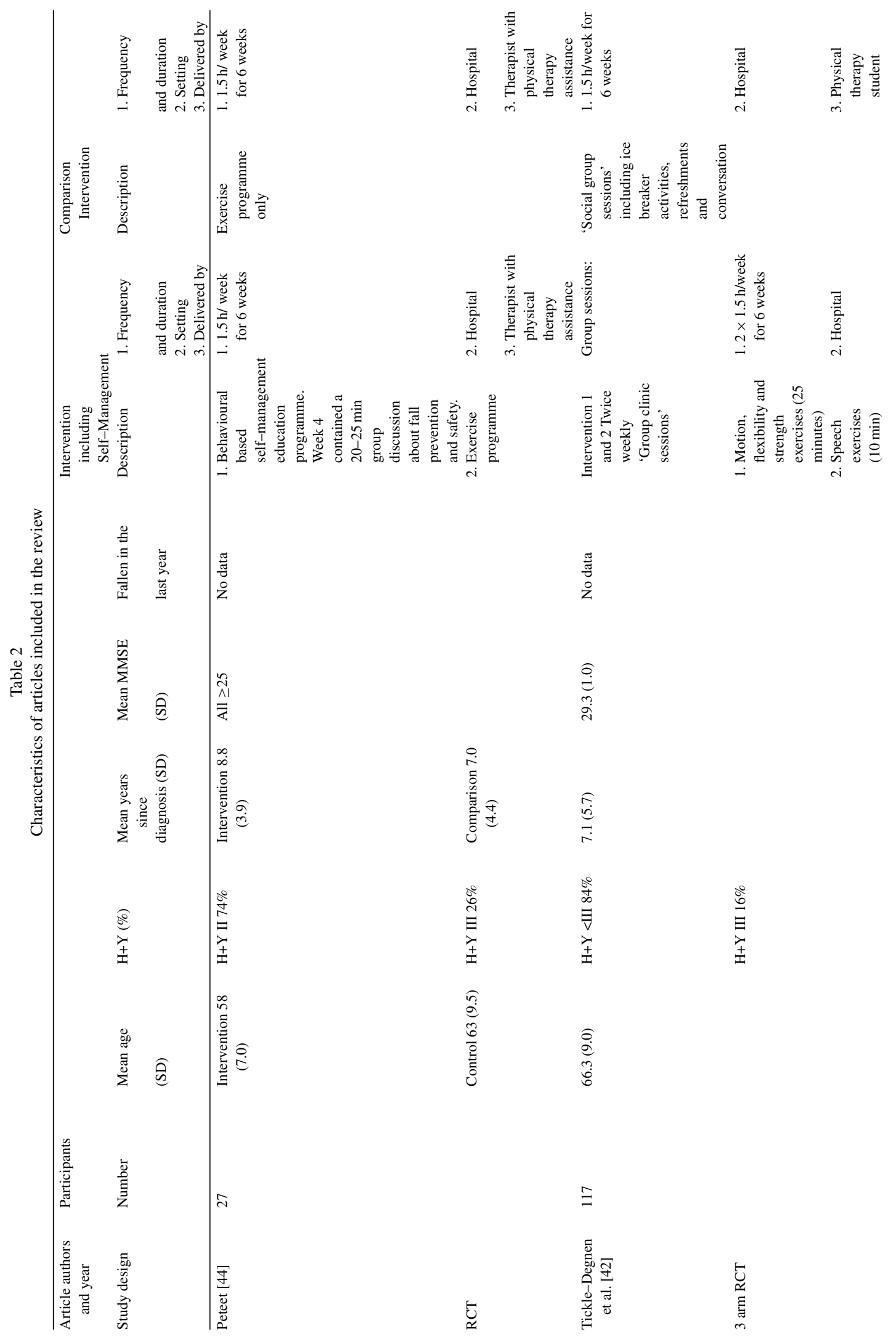




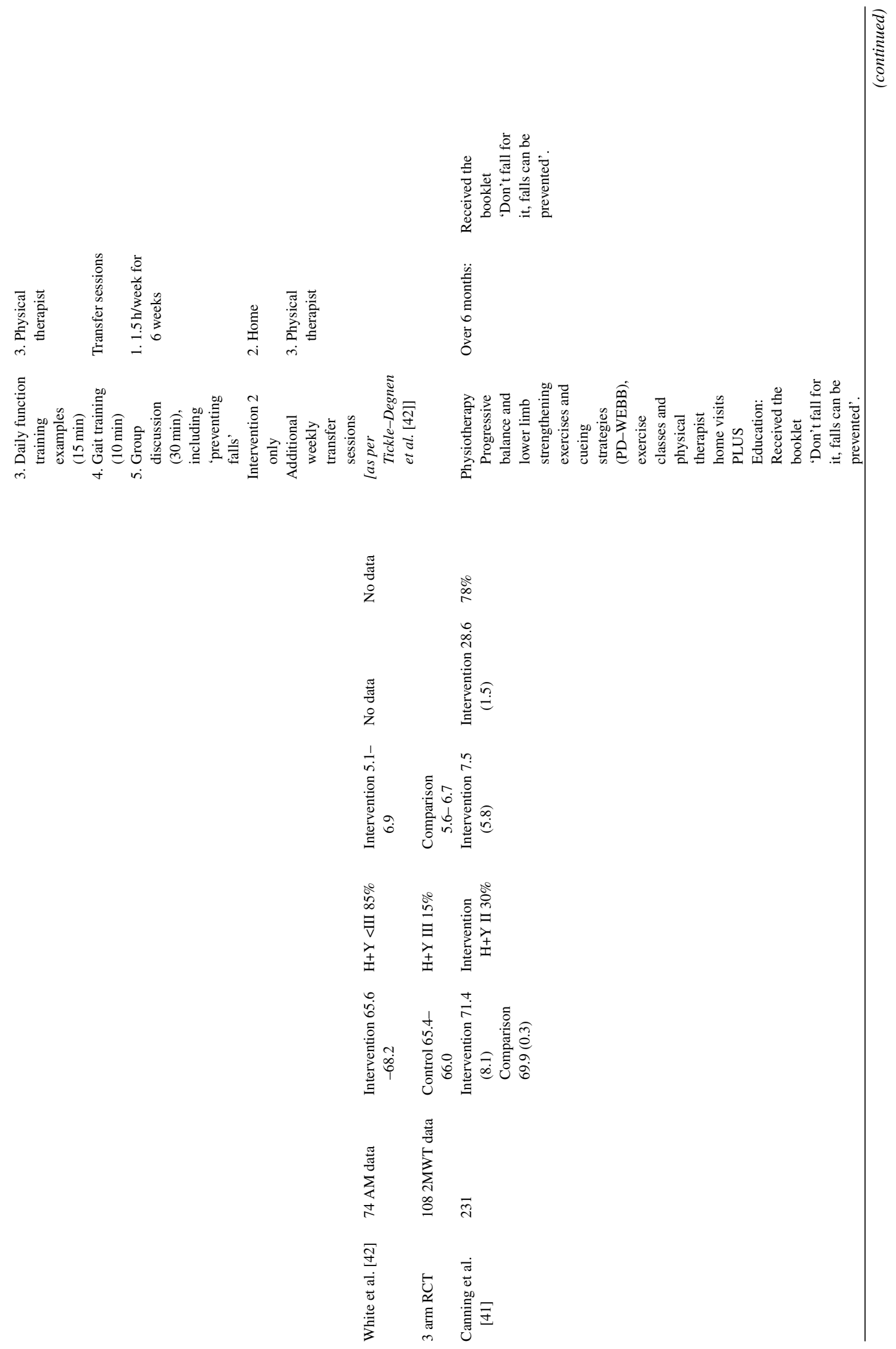




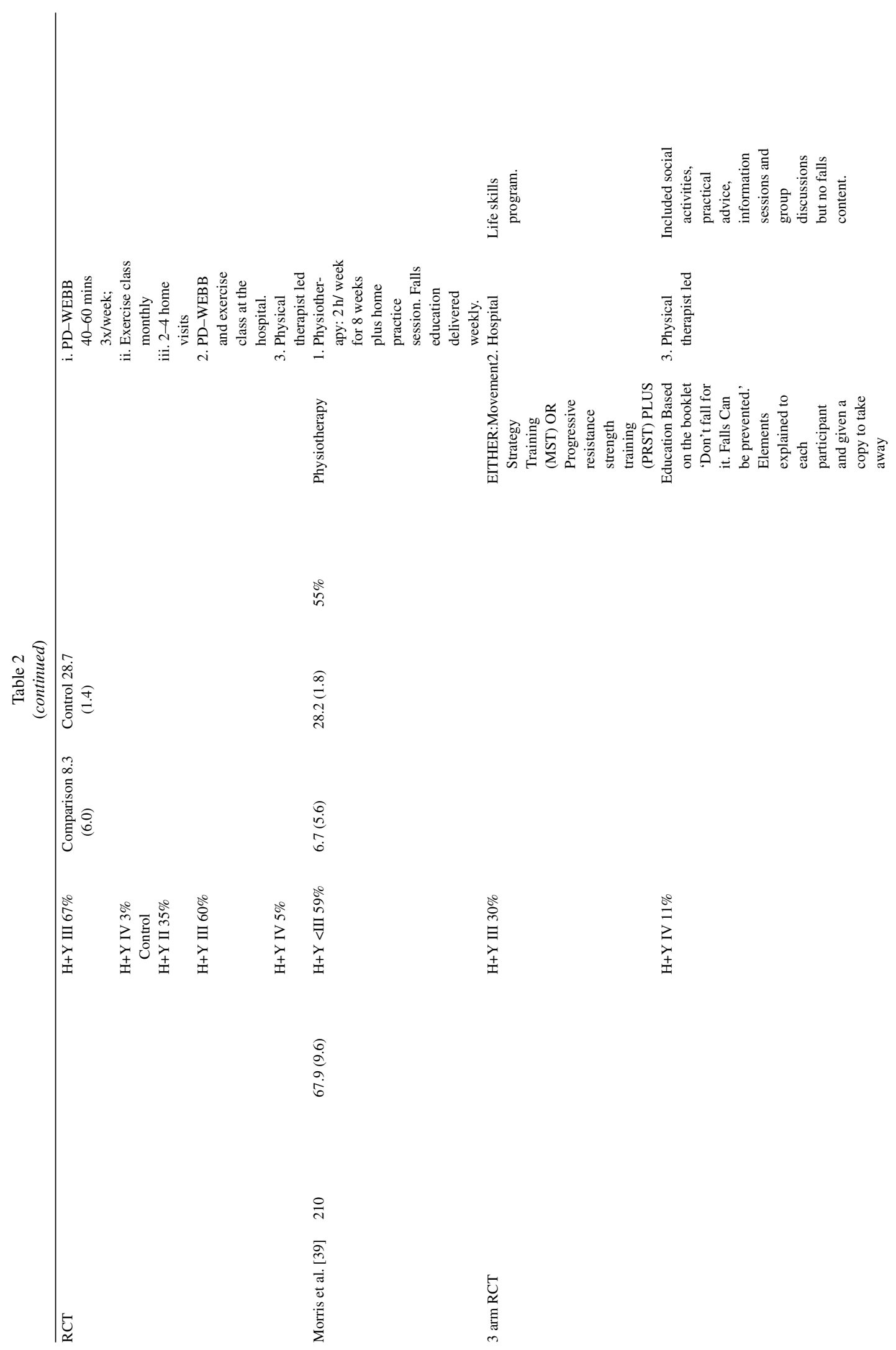




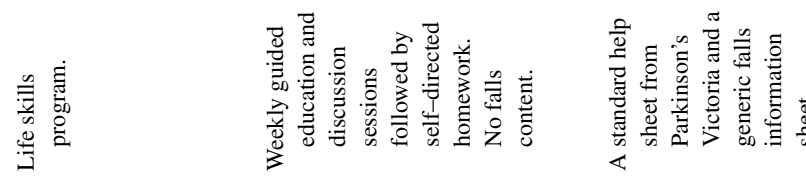

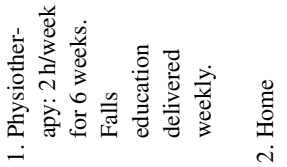

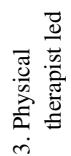

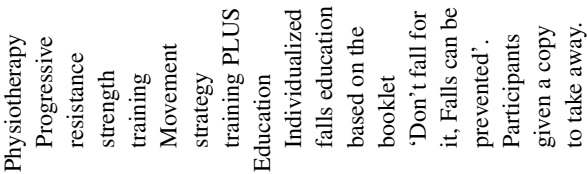

$\stackrel{8}{n}^{2}$

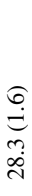

言

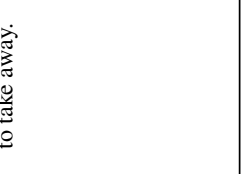

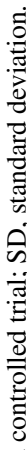

(2)

产

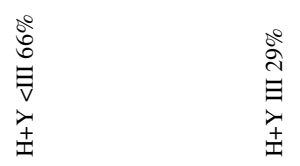

I

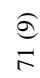

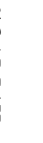

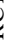

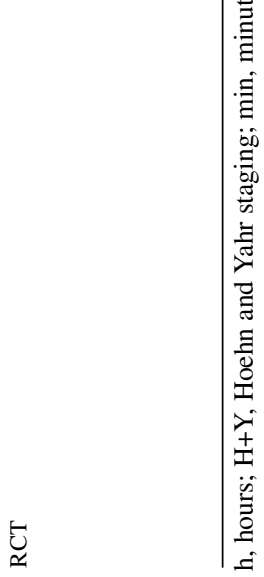




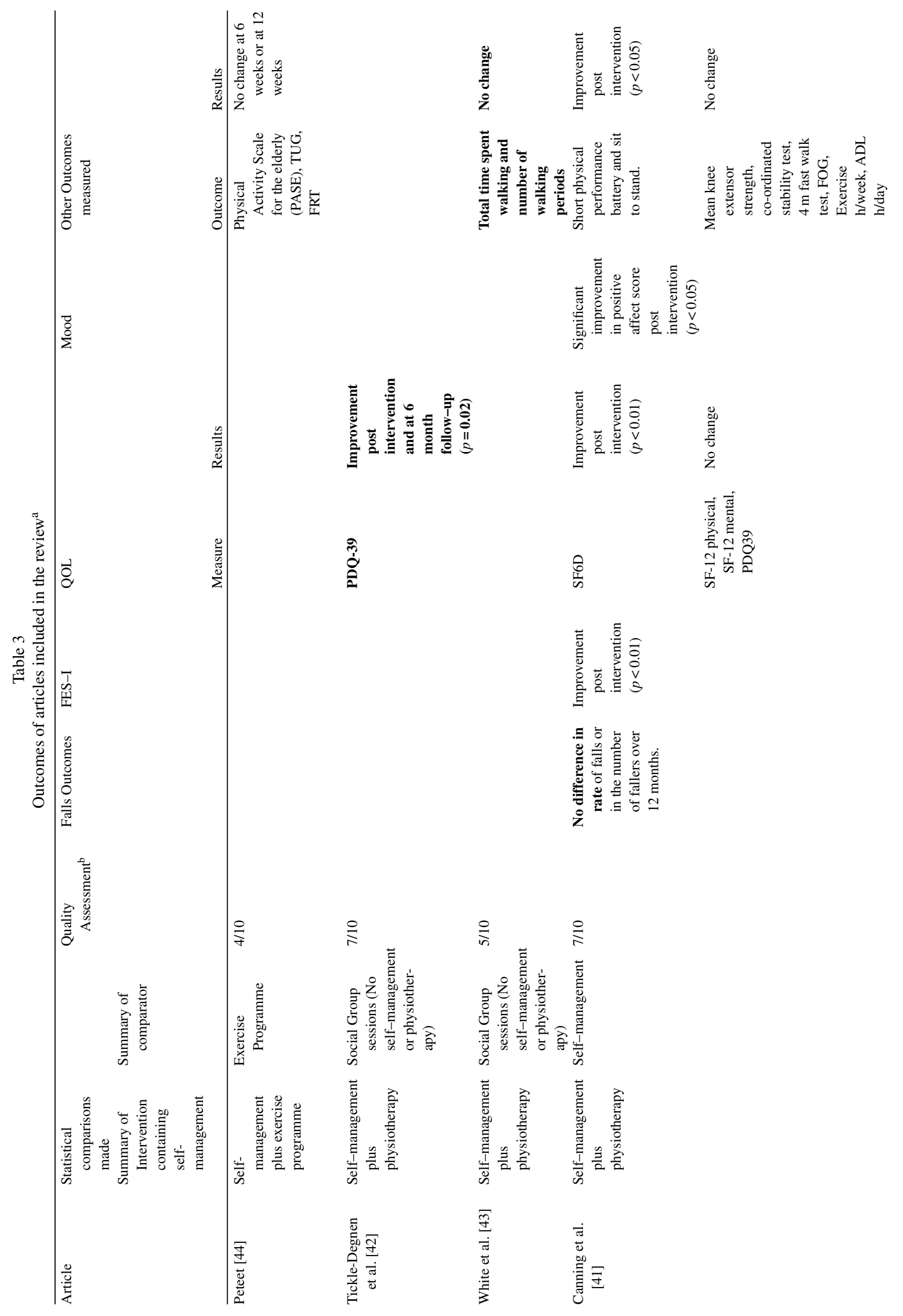




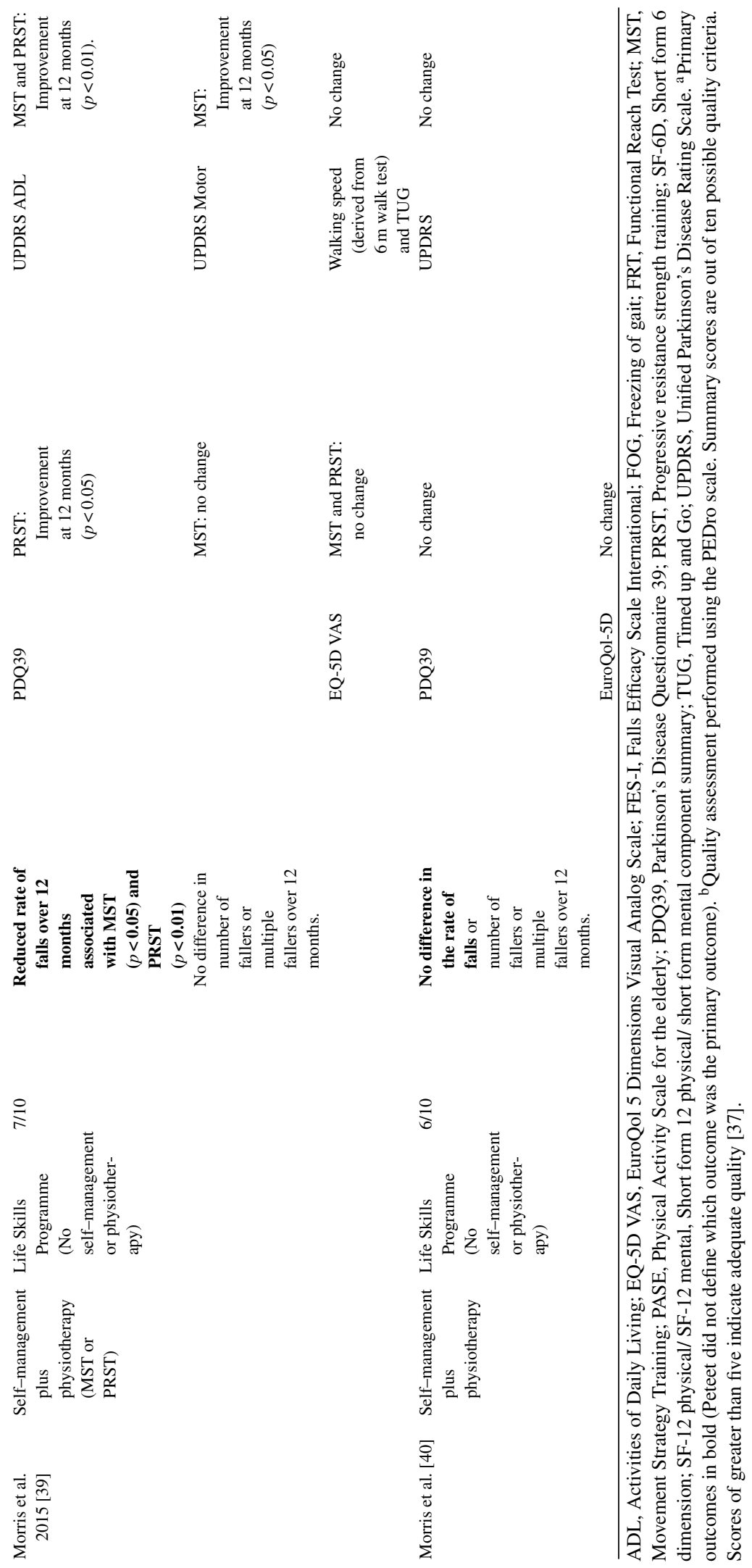


intervention was delivered over six weeks by a physical therapist and contained weekly individualized education sessions. Only week four of the intervention focused on falls, where a 20-25 minute therapist led discussion on falls was delivered, which included falls prevention, safety and strategies to minimize falls. Participants were encouraged to develop and complete exercise-related action plans. TickleDegnen et al. and White et al. assessed the effect of a weekly group-based self-management rehabilitation programme delivered over six weeks by a physical therapist, compared to social group sessions that contained no self-management or falls-based content $[42,43]$. Each session consisted of one hour of physiotherapy and speech therapy followed by a 30 minute group discussion, which included the topics 'preventing falls', 'strategies to improve walking', 'relaxation, stress management' and 'benefits of exercise'.

\section{Falls booklets}

Three articles, Canning et al., Morris et al. 2015 and Morris et al. 2017, contained a self-management component based upon the same the falls booklet 'Don't fall for it. Falls can be prevented!', which was delivered alongside physiotherapy [39-41, 45]. This 32 page booklet is aimed toward all older people at risk of falling and contains no PD-specific information [39-41, 45]. In Canning et al., participants in both arms of the study were given a copy of the booklet and no verbal falls education was delivered; only the physiotherapy component differed between the intervention and control arms [41]. In Morris et al. 2015 over eight weeks, and Morris et al. 2017 over six weeks, weekly falls education was delivered by a physical therapist based upon the content of the booklet and participants were given a copy to take away [39, 40]. In Morris et al. 2015 education was delivered over eight weeks; it was unclear whether it was individualized. In Morris et al. 2017 education was delivered over six weeks and was individualised to participants. The comparator groups in both Morris et al. 2015 and Morris et al. 2017 took part in weekly non-falls education sessions; in Morris et al. 2017 participants also received a non-self-management-based falls information sheet to take away [40].

\section{Analysis of self-management components of the interventions}

Table 1 summarises the self-management intervention components reported in each study based on the PRISMS taxonomy. Description of the self- management component was very brief; articles described them in an average of 60 words [39-43]. Additional information was received from three authors [39, 40, 44]. Through the citations provided, we were able to obtain and directly review the falls booklet utilized within three of the articles [39-41, 45].

\section{Information about the condition and/or its management}

A fundamental part of self-management is arming patients with appropriate information about the condition and/or its management. This component was present in four of six articles. However, the topics, amount and presentation of information differed. Peteet provided participants in both trial arms with written information about PD, with those in the intervention arm also receiving information about falls [44]. In Tickle-Degnen et al and White et al, it is unclear whether participants received any information specific to PD or to falling [42, 43]. The booklet utilized within three articles covers a wide breadth of falls-related topics, including potential aetiologies and different management strategies [45].

\section{Training/rehearsal for everyday activities}

Another key component of a self-management intervention is learning and practicing behaviors and skills that are relevant for living with the condition. All articles delivered this component through physiotherapy strategies such as training with transfers, cueing strategies and movement strategy training, which includes training for functional tasks [39-44]. Five articles delivered this component through self-management strategies. Although the group discussions delivered by Tickle-Degnen et al and White et al, contained information about day to day activities such as strategies to improve dressing and walking, it is unclear whether these were falls-related [42, 43]. The booklet used within three articles encourages users to ask for help from others if they consider a task to be high risk and to plan ahead [45].

\section{Training/rehearsal for psychological strategies}

Self-management interventions often facilitate the development of psychological strategies such as the ability to re-frame symptoms, problem-solve and set personal goals. This component was present in all articles reviewed. The interventions that contained group discussion (Peteet et al., Tickle-Degnen et al. 
and White et al.) taught and encouraged participants to problem-solve and to develop realistic action plans in collaboration with a physical therapist [42-44]. However, it is unclear whether these action plans were falls-related, with no details provided surrounding their implementation. The booklet used within three articles has a page to develop action plan, but the interventions based upon this booklet did not appear to provide participants with training to complete these $[39-41,45]$.

\section{Lifestyle advice and support}

Self-management interventions often contain advice about health and lifestyle; this was included within five of six of the articles. In the group sessions within Tickle-Degnen et al. and White et al., participants discussed the 'Benefits of exercise' [42, 43]. The booklet utilized within three articles promotes a healthy lifestyle, including the importance of physical activity and dietary advice [39-41, 45].

\section{Social support}

All three articles that provided group discussion led by physical therapist provided social support [42-44]. Peteet actively encouraged group interaction; participants were asked to identify a 'buddy' for support although this appeared to be for exercise promotion and not for falls management [44]. No social support component was provided in the three articles that utilized the falls booklet [39-41].

\section{Other components}

Self-management interventions may contain regular clinical review and monitoring of the participant's condition with feedback. Whilst provided within Peteet, these components were targeted toward exercise and not falling [44]. In additional to the components described above, the booklet utilized within three articles supports falls self-management through providing (i) information about available resources, (ii) training to communicate with healthcare professionals to facilitate shared decision-making, and (iii) training/rehearsal for practical self-management through teaching common strategies to reduce fall risk in the home [39-41, 45].

Further components that the PRISMS taxonomy suggests may be contained within a self-management intervention, but were not included within any of the articles, were (i) individualized action plans prepared by a healthcare professional, (ii) practical support with adherence, (iii) provision of equip- ment, and (iv) easy access to further advice and support.

\section{Outcome measures}

All articles assessed the effect of the intervention as a whole (Table 3). No articles separated the effect of the self-management component from the physiotherapy component, or assessed skill acquisition or adherence to the self-management component.

\section{Falls outcomes}

The three articles where the self-management component was based upon the falls booklet reported falls [39-41]. In Morris et al. 2015 participants fell less over 12 months when they received physiotherapy and self-management versus no physiotherapy or self-management [39]. However, no improvement was seen in the total number of participants who fell in Canning et al., Morris et al. 2015 or Morris et al. 2017 [39-41]. Morris et al. 2015 and Morris et al. 2017 found no difference in the number of multiple fallers, with combined physiotherapy and self-management versus no physiotherapy or self-management [39, 40]. Sub analysis in Canning et al. found a 69\% reduction in falls in those with less advanced PD who received physiotherapy and self-management versus self-management alone [41]. Articles based on group discussion did not report falls outcomes.

Only Canning et al. assessed fear of falling, which was measured through the Falls Efficacy Scale- International scale, which assesses concerns about falling in 16 different circumstances [41, 46]. Scores significantly improved at six months in participants who received physiotherapy and self-management versus self-management alone [41].

\section{Quality of life ( $Q O L)$}

Four articles assessed QOL. Tickle-Degnen et al. reported that combined physiotherapy and self-management was associated with significant improvement in health-related QOL (HRQOL) measured by Parkinson's Disease Questionnaire 39 (PDQ39) at programme end at six weeks when compared to the control arm; improvement persisted at six months [42]. The three studies whose self-management component was based upon the falls booklet produced inconsistent results regarding QOL. In Morris et al. 2015, when compared to no physiotherapy or self-management, one intervention arm (progressive resistance and strength training plus self-management) was associated with 
an improvement in QOL as measured by PDQ39 but no change was observed in EuroQol 5 Dimensions Visual Analogue scale (EQ-5D VAS); in the other intervention arm (Movement strategy training plus self-management) no improvements were seen in either PDQ39 or EQ-5D VAS [39]. The combined physiotherapy and self-management intervention in Morris et al. 2017, was not associated with improvements in QOL when compared to no physiotherapy or self-management [40]. Canning reported significant improvement in Short-Form Six-Dimension (SF-6D) in participants who received physiotherapy and selfmanagement compared to self-management alone; however, no change was observed in relation to either PDQ39 or the mental and physical sub scores of Short-Form 12 (SF-12) [41].

\section{Other outcome measures}

Four articles reported various physical activity outcomes [39, 41, 42, 44]. Peteet, Morris et al. 2015 and White et al. found no statistical difference in physical activity outcome measures in participants who received each of the respective physiotherapy and self-management interventions compared to no physiotherapy and self-management [39, 43, 44]. Canning et al. reported improvement in the short physical performance battery and sit to stand in participants who received physiotherapy and self-management compared to self-management alone, but no improvement was seen in a number of other measures including exercise hours/week [41].

Two articles collected Unified Parkinson's disease rating scale (UPDRS) data. Morris et al. 2015 reported significant improvement in the activities of Daily Living (ADL) component of UPDRS, but not the motor component of the UPDRS in participants who received physiotherapy and self-management versus no physiotherapy or self-management [39]. Morris et al. 2017 reported no change in UPDRS with physiotherapy and self-management versus no physiotherapy and self-management [40].

Canning et al. reported adverse outcomes related to the physiotherapy component of the intervention; two participants fell whilst exercising at home [41]. No studies reported adverse outcomes relating to the self-management component.

\section{DISCUSSION}

This is the first attempt to systematically review the literature on falls self-management interventions for PwP. Other reviews have begun to explore more generic self-management interventions to support PwP [29]. Despite a thorough and systematic search, few relevant studies were identified, allowing us to conclude that this is not a well-researched area. All of the articles included studied the effect of a selfmanagement intervention, comprised of either group discussion or a falls booklet, alongside a physiotherapy intervention. Self-management was often not the main focus of the intervention and was incompletely described. Articles displayed considerable variation in outcomes measured.

\section{Methodological comments}

All articles with the exception of Peteet were of adequate quality [44]. However, participants enrolled into studies were not representative of the range of PwP who fall, typically recruiting those with lower Hoehn and Yahr scores for example, and two articles studied the effect of the same intervention on the same cohort of participants. All articles excluded those with cognitive impairment, yet this group are at heightened risk of falling and its negative physical and psychological outcomes [4]. No articles included caregivers, who play a pivotal role in falls management $[4,8]$. Including caregivers in future research programmes may facilitate the inclusion of $\mathrm{PwP}$ with cognitive impairment, and improve caregiver outcomes.

Whilst the articles included a wide breadth of self-management strategies, these were often incompletely described, as has been identified in previous reviews of self-management interventions [34]. It is unclear whether the interventions delivered self-management as planned. Whilst multifaceted interventions may be more likely to benefit older people who fall, all intervention components should be well described to allow for them to be identified and replicated [32, 47-49].

Key attributes that an individual requires to effectively self-manage a LTC are problem-solving, decision-making and goal setting [22]. None of the articles included within this review studied the effect of an intervention that clearly taught and promoted all three of these skills. Individuals also require information specific to their needs; however, in only one article was the intervention individualized to participants $[22,40]$. Providing a heterogeneous population with generic information can lead to information overload and user disengagement [39-41, 45, 50]. Future falls self-management programmes for PwP 
should explore tailoring of information to participants' needs to increase the likelihood of adherence and long-term behavioral change [23, 51].

\section{Impact of self-management programmes on falls and other clinical outcomes}

All articles assessed the effect of physiotherapy and self-management in combination, making it impossible to draw firm conclusions about the specific contribution of falls self-management from the studies conducted so far.

In the three articles where falls outcomes were measured, only one recorded a reduced rate of falls, and none showed a reduction in the number of fallers [39, 41, 52]. Given that current evidence for rehabilitation interventions to reduce falls is inconclusive, the effect of self-management on falls remains unclear. Only Canning et al. 2015 measured fear of falling, however, as only the physiotherapy component differed between the intervention and comparator groups, the effect of self-management on fear of falling remains unexplored [41].

Most articles measured QOL but only the intervention studied by Tickle-Degnen et al., which contained group discussion and education to problem-solve and develop action plans, led to an improvement [42]. Group-based self-management programmes have previously been shown to provide social support and psychological benefit [53]. However, future researchers should consider their acceptability to participants with reduced mobility, where travel may be difficult, and explore the impact of programme end, which can lead to feelings of isolation [53].

No articles measured self-management outcomes such as applicable knowledge or independence, which are considered important by those with LTCs [54]. It is impossible to tell whether and to what extent PwP engaged with the self-management components. Future self-management programmes should look to include process evaluation measures such as acceptability, adherence and engagement; mixed methods are likely to be useful [55].

\section{Conclusion}

Overall, the scarcity of published research, plus the nature of the studies to date, have made it impossible to draw any firm conclusions about the overall efficacy of falls self-management interventions for PwP, or to identify the important components of these interventions.
Within the articles included in this review, it is unclear whether interventions were targeted toward $\mathrm{PwP}$ who had experienced falls, and information was rarely individualized to participants. Future studies of programmes of falls self-management interventions may benefit from targeting those whom falls management is most critical, to include PwP with a history of falls, fear of falling and/or cognitive impairment, and caregivers.

Given the limitations of other interventions to support falling in PwP, and the benefits seen in selfmanagement programmes in other LTCs, it is important that effective falls self-management programmes are developed and evaluated to support PwP.

\section{ACKNOWLEDGMENTS}

We thank Paula Sands, Health Services librarian, and Dr Emma Stack from the University of Southampton for their support.

$\mathrm{CO}$ was supported by the University of Southampton NIHR Academic Clinical Fellow (ACF) training programme. HCR is supported by the NIHR Southampton Biomedical Research Centre. CO, KI and HCR are supported by NIHR CLAHRC Wessex. This is a summary of research conducted by the National Institute for Health Research (NIHR) Collaboration for Leadership in Applied Health Research and Care (CLAHRC) Wessex at the University of Southampton. The views of those expressed are those of the authors and not necessarily those of the NHS, NIHR, or the Department of Health.

\section{CONFLICT OF INTEREST}

The Authors have no conflict of interest to report.

\section{SUPPLEMENTARY MATERIAL}

The supplementary material is available in the electronic version of this article: http://dx.doi.org/ 10.3233/JPD-181524.

\section{REFERENCES}

[1] Wood BH, Bilclough JA, Bowron A, Walker RW (2002) Incidence and prediction of falls in Parkinson's disease: A prospective multidisciplinary study. J Neurol Neurosurg Psychiatry 72, 721-725.

[2] Hiorth YH, Larsen JP, Lode K, Tysnes O-B, Godfrey A, Lord S, Rochester L, Pedersen KF (2016) Impact of falls on physical activity in people with Parkinson's disease. J Parkinsons Dis 6, 175-182. 
[3] Lindholm B, Hagell P, Hansson O, Nilsson MH (2014) Factors associated with fear of falling in people with Parkinson's disease. BMC Neurol 14, 19.

[4] Amar K, Stack E, Fitton C, Ashburn A, Roberts HC (2015) Fall frequency, predicting falls and participating in falls research: Similarities among people with Parkinson's disease with and without cognitive impairment. Parkinsonism Relat Disord 21, 55-60.

[5] van der Marck MA, Klok MPC, Okun MS, Giladi N, Munneke M, Bloem BR (2014) Consensus-based clinical practice recommendations for the examination and management of falls in patients with Parkinson's disease. Parkinsonism Relat Disord 20, 360-369.

[6] Muslimovic D, Post B, Speelman JD, Schmand B (2005) Cognitive profile of patients with newly diagnosed Parkinson disease. Neurology 65, 1239-1245.

[7] Hely MA, Reid WGJ, Adena MA, Halliday GM, Morris JGL (2008) The Sydney multicenter study of Parkinson's disease: The inevitability of dementia at 20 years. Mov Disord 23, 837-844.

[8] McLaughlin D, Hasson F, Kernohan WG, Waldron M, McLaughlin M, Cochrane B, Chambers H (2011) Living and coping with Parkinson's disease: Perceptions of informal carers. Palliat Med 25, 177-182.

[9] Dow B, Meyer C, Moore K, Hill K (2013) The impact of care recipient falls on caregivers. Aust Health Rev 37, 152-157.

[10] Davey C, Wiles R, Ashburn A, Murphy C (2004) Falling in Parkinson's disease: The impact on informal caregivers. Disabil Rehabil 26, 1360-1366.

[11] Tomlinson CL, Patel S, Meek C, Clarke CE, Stowe R, Shah L, Sackley CM, Deane KHO, Herd CP, Wheatley K, Ives N (2012) Physiotherapy versus placebo or no intervention in Parkinson's disease. Cochrane Database Syst Rev, CD002817.

[12] Shen X, Wong-Yu ISK, Mak MKY (2016) Effects of exercise on falls, balance, and gait ability in Parkinson's disease. Neurorehabil Neural Repair 30, 512-527.

[13] Allen NE, Sherrington C, Paul SS, Canning CG (2011) Balance and falls in Parkinson's disease: A meta-analysis of the effect of exercise and motor training. Mov Disord 26, 1605-1615.

[14] Van de Weijer SCF, Hommel ALAJ, Bloem BR, Nonnekes J, De Vries NM (2018) Promising non-pharmacological therapies in PD: Targeting late stage disease and the role of computer based cognitive training. Parkinsonism Relat Disord 46, S42-S46.

[15] Lamb SE, Mistry D, Alleyne S, Atherton N, Brown D, Copsey B, Dosanjh S, Finnegan S, Fordham B, Griffiths F, Hennings S, Khan I, Khan K, Lall R, Lyle S, Nichols V, Petrou S, Zeh P, Sheehan B (2018) Aerobic and strength training exercise programme for cognitive impairment in people with mild to moderate dementia: The DAPA RCT. Health Technol Assess (Rockv) 22, 1-202.

[16] Rao AK (2010) Enabling functional independence in Parkinson's disease: Update on occupational therapy intervention. Mov Disord 25, S146-S151.

[17] Clarke CE, Patel S, Ives N, Rick CE, Woolley R, Wheatley K, Walker MF, Zhu S, Kandiyali R, Yao G, Sackley CM (2016) Clinical effectiveness and cost-effectiveness of physiotherapy and occupational therapy versus no therapy in mild to moderate Parkinson's disease: A large pragmatic randomised controlled trial (PD REHAB). Health Technol Assess (Rockv) 20, 1-96.
[18] Sturkenboom IHWM, Graff MJL, Hendriks JCM, Veenhuizen Y, Munneke M, Bloem BR, Nijhuis-van der Sanden MW, OTiP study group (2014) Efficacy of occupational therapy for patients with Parkinson's disease: A randomised controlled trial. Lancet Neurol 13, 557-566.

[19] van der Eijk M, Faber MJ, Al Shamma S, Munneke M, Bloem BR (2011) Moving towards patient-centered healthcare for patients with Parkinson's disease. Parkinsonism Relat Disord 17, 360-364.

[20] Grosset KA, Grosset DG (2005) Patient-perceived involvement and satisfaction in Parkinson's disease: Effect on therapy decisions and quality of life. Mov Disord 20, 616619.

[21] Self-management programme | Parkinson's UK. https://www.parkinsons.org.uk/information-and-support/ self-management-programme. Accessed on 12 October 2018.

[22] Lorig KR, Holman H (2003) Self-management education: History, definition, outcomes, and mechanisms. Ann Behav Med 26, 1-7.

[23] Foster G, Taylor SJC, Eldridge SE, Ramsay J, Griffiths CJ (2007) Self-management education programmes by lay leaders for people with chronic conditions. Cochrane Database Syst Rev, CD005108.

[24] Fryer CE, Luker JA, McDonnell MN, Hillier SL (2016) Self management programmes for quality of life in people with stroke. Cochrane Database Syst Rev, CD010442.

[25] Zwerink M, Brusse-Keizer M, van der Valk PDLPM, Zielhuis GA, Monninkhof EM, van der Palen J, Frith PA, Effing T (2014) Self management for patients with chronic obstructive pulmonary disease. Cochrane Database Syst Rev, CD002990.

[26] Pal K, Eastwood S V, Michie S, Farmer AJ, Barnard ML, Peacock R, Wood B, Inniss JD, Murray E (2013) Computerbased diabetes self-management interventions for adults with type 2 diabetes mellitus. Cochrane Database Syst Rev, CD008776.

[27] Steinsbekk A, Rygg L, Lisulo M, Rise MB, Fretheim A (2012) Group based diabetes self-management education compared to routine treatment for people with type 2 diabetes mellitus. A systematic review with meta-analysis. BMC Health Serv Res 12, 213.

[28] van der Eijk M, Nijhuis FAP, Faber MJ, Bloem BR (2013) Moving from physician-centered care towards patientcentered care for Parkinson's disease patients. Parkinsonism Relat Disord 19, 923-927.

[29] Kessler D, Liddy C (2017) Self-management support programs for persons with Parkinson's disease: An integrative review. Patient Educ Couns 100, 1787-1795.

[30] Zwar N, Harris M, Griffiths R, Martin R, Sarah D, Davies G, Hasan I (2006) A systematic review of chronic disease management. Australian Primary Health Care Research Institute.

[31] Moher D, Liberati A, Tetzlaff J, Altman DG, Altman D (2009) Preferred reporting items for systematic reviews and meta-analyses: The PRISMA Statement. PLoS Med 6, e1000097.

[32] Craig P, Dieppe P, Macintyre S, Michie S, Nazareth I, Petticrew M (2013) Developing and evaluating complex interventions: The new Medical Research Council guidance. Int J Nurs Stud 50, 587-592.

[33] Pearce G, Parke HL, Pinnock H, Epiphaniou E, Bourne C LA, Sheikh A, Taylor SJ (2016) The PRISMS taxonomy of self-management support: Derivation of a novel taxonomy 
and initial testing of its utility. J Health Serv Res Policy 21, 73-82.

[34] Connor M, Norman P, eds. (2015) Predicting and Changing Heath Behaviour, McGraw Hill Education, pp. 358-389.

[35] Hoffmann TC, Erueti C, Glasziou PP (2013) Poor description of non-pharmacological interventions: Analysis of consecutive sample of randomised trials. BMJ 347, f3755.

[36] Physiotherapy Evidence Database (PEDro) scale (English). https://www.pedro.org.au/english/downloads/pedro-scale. Last updated June 21 1999, Accessed on January 112019.

[37] Armijo-Olivo S, da Costa BR, Cummings GG, Ha C, Fuentes J, Saltaji H, Egger M (2015) PEDro or Cochrane to assess the quality of clinical trials? A meta-epidemiological study. PLoS One 10, e0132634.

[38] Caunter M, Grantier L (2010) Development and utilization of a falls prevention booklet for people with Parkinson's. Parkinsonism Relat Disord 16, S80.

[39] Morris ME, Menz HB, McGinley JL, Watts JJ, Huxham FE, Murphy AT, Danoudis ME, Iansek R (2015) A randomized controlled trial to reduce falls in people with Parkinson's disease. Neurorehabil Neural Repair 29, 777-785.

[40] Morris ME, Taylor NF, Watts JJ, Evans A, Horne M, Kempster P, Danoudis M, McGinley J, Martin C, Menz HB (2017) A home program of strength training, movement strategy training and education did not prevent falls in people with Parkinson's disease: A randomised trial. J Physiother 63, 94-100.

[41] Canning CG, Sherrington C, Lord SR, Close JCT, Heritier S, Heller GZ, Howard K, Allen NE, Latt MD, Murray SM, O'Rourke SD, Paul SS, Song J, Fung VSC (2015) Exercise for falls prevention in Parkinson disease: A randomized controlled trial. Neurology 84, 304-312.

[42] Tickle-Degnen L, Ellis T, Saint-Hilaire MH, Thomas CA, Wagenaar RC (2010) Self-management rehabilitation and health-related quality of life in Parkinson's disease: A randomized controlled trial. Mov Disord 25, 194-204.

[43] White D, Wagenaar R, Ellis T, Tickle-Degnen L (2009) Changes in walking activity and endurance following rehabilitation for people with Parkinson's disease. Arch Phys Med Rehabil 90, 43-50.

[44] Peteet JO (2002) Self-management of Parkinson's disease: The effect of a group exercise program on lifestyle physical activity, self-efficacy, and function.
[45] Commonwealth of Australia (2007) Don't Fall for It. Falls Can be Prevented! A Guide to Preventing Falls for Older People, Australian Government Publisher.

[46] Yardley L, Beyer N, Hauer K, Kempen G, Piot-Ziegler C, Todd C (2005) Development and initial validation of the Falls Efficacy Scale-International (FES-I). Age Ageing 34, 614-619.

[47] CDC (2004) Improving the reporting quality of nonrandomized evaluations of behavioral and public health interventions: The TREND Statement. Am J Public Health 94, 361-366.

[48] Schulz KF, Altman DG, Moher D, CONSORT Group (2010) CONSORT 2010 statement: Updated guidelines for reporting parallel group randomised trials. BMJ 340, c332.

[49] Gillespie LD, Robertson MC, Gillespie WJ, Sherrington C, Gates S, Clemson LM, Lamb SE (2012) Interventions for preventing falls in older people living in the community. In Cochrane Database of Systematic Reviews, Gillespie LD, ed. John Wiley \& Sons, Ltd, Chichester, UK.

[50] Bawden D, Robinson L (2009) The dark side of information: Overload, anxiety and other paradoxes and pathologies. J Inf Sci 35, 180-191.

[51] Kreuter MW, Strecher VJ, Glassman B (1999) One size does not fit all: The case for tailoring print materials. Ann Behav Med 21, 276-283.

[52] Morris R, Lord S, Lawson RA, Coleman S, Galna B, Duncan GW, Khoo TK, Yarnall AJ, Burn DJ, Rochester L (2017) Gait rather than cognition predicts decline in specific cognitive domains in early Parkinson's disease. J Gerontol A Biol Sci Med Sci 71, 1616-1623.

[53] Wilson PM, Kendall S, Brooks F (2007) The Expert Patients Programme: A paradox of patient empowerment and medical dominance. Health Soc Care Community 15, 426-438.

[54] Boger E, Ellis J, Latter S, Foster C, Kennedy A, Jones F, Fenerty V, Kellar I, Demain S (2015) Self-management and self-management support outcomes: A systematic review and mixed research synthesis of stakeholder views. PLoS One 10, $\mathrm{e} 0130990$.

[55] Moore GF, Audrey S, Barker M, Bond L, Bonell C, Hardeman W, Moore L, O'Cathain A, Tinati T, Wight D, Baird J (2015) Process evaluation of complex interventions: Medical Research Council guidance. BMJ 350, h1258. 